\section{NHS quality improvement could be driven by less formal regulation}

In 2019 NHS Improvement and NHS England merged, becoming one major organisation responsible for regulating and improving the of care and patient safety without increasing costs, is under debate. A comprehensive evaluation of a five year partnership between the English NHS and Virginia Mason Institute in the USA finds a less formal, more relational approach to governance produces a reciprocal learning environment for both regulators and service providers. Dr Nicola Burgess and colleagues from Warwick Business School (University of Warwick), suggest a relational approach to governance is vital for the development of a sustainable continuous improvement culture in a healthcare context.

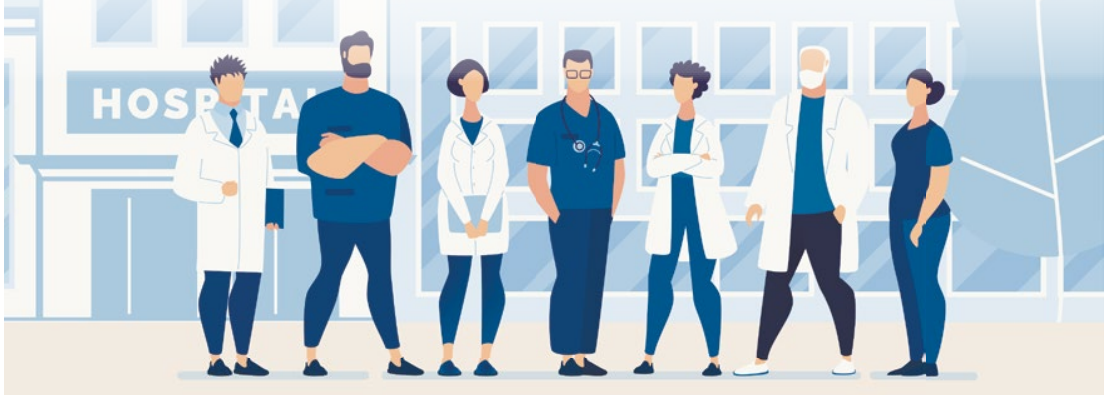

ealthcare regulatory organisations hierarchy, issuing commands to the sevice providers (NHS Trusts, hospitals) below them in the pecking order. But this top-down regulation can lead to providers focusing on implementing new processes or meeting targets, and not on whether the new regulation has any value to customers (usually patients). Since the merging of NHS England and hHS Improvement in 2019 there has benlabre about how this new major hospitals to foster a sustainable culture of continuous improvement capability

The Virginia Mason Production System

(NMPS) is a translation of the Toyota

Production System, widely known as

Lean. The Toyota Production System

evolved from the late 1940 s to compete

that had invested heavily in automation

to achieve economies of scale through

mass manufacturing. At the time, Toyota

had a very poor reputation for quality and

very litte money to invest in machinery

rolued to produce cars at the same

of World War II Japanese industry faced

severe capacity constraints with a reduced

workforce and a scarcity of raw materials.

So it follows that Toyota had to find a

way to compete, using less of everything.

Their goal was achieved through adopting an uncompromising focus on 'value' from the perspective of the customer, and to make value-adding activities flow seamlessly without any waste.

Dr Nicola Burgess and colleagues from Warwick Business School (University of Warwick) have been analysing the (S) Masoen the English NHS and the Virg Mason Institute (Seattle, USA). The goal of the partnership was to transfer learning from an adaption of the Toyota Production System (also known as Lean) hospital in Seattle - to five English
Implemented as a production system, Lean requires everyone's involvement in the pursult of continuous improvement through elimination of activities and collectively as 'waste'. Applied to processes that don't add value from

\section{THE VIRGINIA MASON}

with American car manufacturers like Ford

manufact the perspective of the customer. These healthcare, the goal of a production system is to continuously elminate care deliver is continuouly imper of benefitting both patient and payer since less resource is spent on activities that deliver no value to the patient.

Improving the quality of patient care while simultaneously reducing the cost of care delivery is an attractive proposition for healthcare leaders. Many healthcare organisations around the world have attempted to integrate Lean production principles into their operation, but very few have success long-term or on a large scale. Difficulties come in many forms, from lack of internal improvement capability, professional resistance towards mprovement methods, and pressure the common to the highly regulted professional envirinmegulaupes

opportunity for creativity in developing solutions to complex problems and thereby improving performance of

complex systems.

\section{NHS PARTNERSHIP FOR INTER-} ORGANISATIONALLEARNING

The Virginia Mason Medical Center, a hospital in Seattle, is a notable exception to the widespread failure to effectively implement Lean principles; in fact, they have been successfully enacting the system since 2002. The Virginia Mason production System puts patients and the of care at the centre of their of care at the centre of their operating Virginia Mason Medical Center gaining reputation as one of the safest hospital in the world.

In 2015 five English NHS hospitals were selected to form a partnership with the Virginia Mason Institute and implement localised versions of the Virginia Mason Production System. The goal of the partnership is for each of the hospitals to develop localised versions of the production system and foster a sustainable culture of conthous improvement capability in their organisations

Since 2018, Dr Nicola Burgess and colleagues at Warwick Business School have been leading a comprehensive

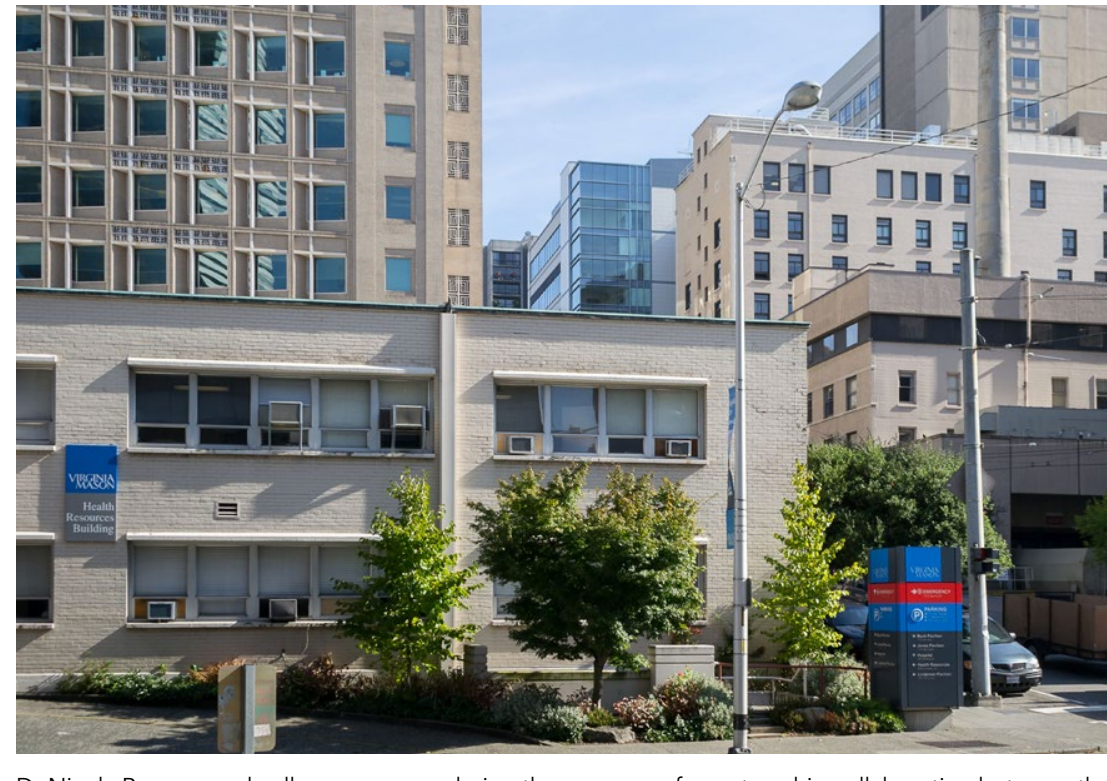

Dr Nicola Burgess and colleagues are analysing the progress of a partnership collaboration between the
English NHS Medical Center the provides education, training and exeecutive coaching in the Virginia Mason Production System to other

evaluation of the partnership between the NHS hospitals and the Virginia Mason Institute. The team are due to produce a full report in 2021, but their observations so far have found positive results. One unexpected, but promising finding illustrates a shift in the relationships between senior members

Through this regular meeting, a partnership 'spirit' has emerged between senior members of NHS England/NHS mprovement, Virginia Mason Institute and the five CEOs. The meeting requires each CEO to present their progress with systems, the results of improvement work, and reflections on challenges

A protected relational space refers to a safe and supportive atmosphere... where collaborators should feel free to discuss both their successes and failures without judgement.

and the CEOs of each of the five NHS hospitals. This shift can be credited to the partnership with the Virginia Mason Institute and a commitment to the Production System.

A PROMISING START During the evaluation, Dr Burgess and her colleagues discovered a significant shift in the inter-organisational relationships as a result of the partnership. One particul aspect of the implementation of the found to be particurly system was found to be particularly beneficial: the monthly meeting of a Tridance Board (TGB). experienced. While the meeting is clearly structured for governance and accountability, conversations are frank and fluid between the peer group of CEOs 列 NHS Improvement. The monthly meeting where new knowledge is co-created and all members are learning together.

So what are the key factors for making this parthership successful? Dr Burgess and colleagues have identified two parnership protected relational spact partnership. protected relational space known as a 'compact'. 


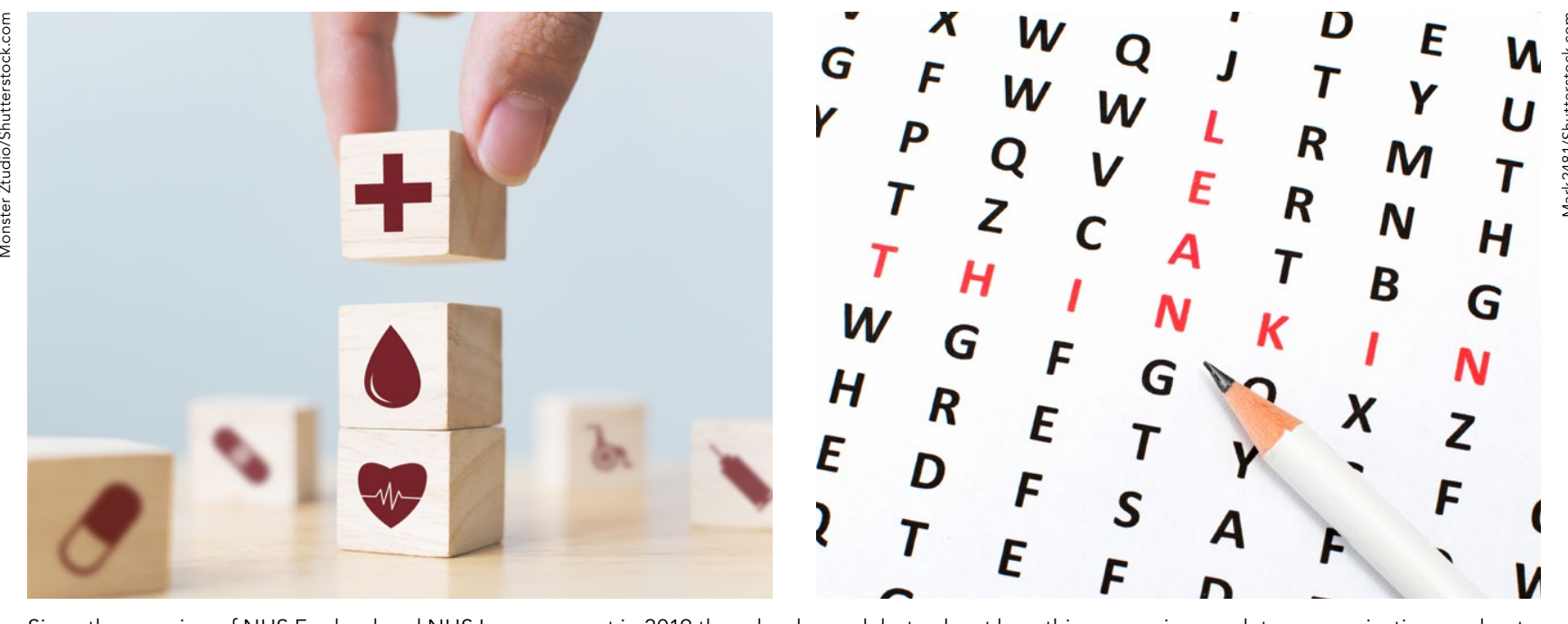

Since the merging of NHS England and NHS Improvement in 2019 there has been deb
foster a culture of continuous improvement across the healthcare system as a whole

A PROTECTED RELATIONAL SPACE A protected relational space refers to a than a specific should feel free to discuss both their successes and failures without judgement. This should also be a space where processes can be discussed that would challenge current institutional practises.

The monthly face-to-face meeting of the Transformation Guidance Board provides an example of a protected relational space. The monthly meeting would last around six hours and did not feature any phones, laptops or other managerial distractions. Contrary to what might be expected, several representatives described these meetings as "the best day of the month". The CEOs from the meeting Dr Burgess and collegus attribute this attitude to the friendly informal atmosphere that characterises these meetings, and allows for the frank discussion of any and all issues fostered honest discussions and built
trusting relationships between senior trusting relationships bet
partnership members.

\section{THE COMPACT}

A compact is an informal, non-binding in the partnership, akin to an explicit (mutually agreed) psychological contract. This might be considered a first step towards building relational authority, as the representatives must work together to agree on the terms of the compact. The members of the NHS partnership took nearly 12 months to negotiate the exp outlined in the compact.

An important behavioural aspect of the compact was an agreement to with related responsilities induding listening, and piving and receiving constructive criticism with good intent (or the assumption of good intent on the part of the collaborator giving the

positive atmosphere of the collaboration and being empathetic with issues ncountered by service providers as hey foster localised versions of the external pressure to demonstrate immediate change.

There were many factors considered in the development of the compact, all of which form the guidelines for how the collaboration should proceed. If then on group does not meet the terms of the compact, an open discussion takes place at the monthly meeting of the TGB to make sense of the breach and consider what all members of the collaboration could do differently to improve progress

\section{IMPROVEMENT IN THE NHS}

Dr Burgess and colleagues suggest that this relational approach to regulation

wider NHS system. The positive attitudes

. The positive attitudes in the partnership the collaborators. Over time, the relationship between

Dr Burgess and colleagues suggest that this relational approach to regulation and the shitt to regulator and service provider shifted from one

characterised could and should be extended to the open and honest
discussion and earning between hroups, suggest this could be a wider NHS system.

criticism). Another behavioural factor considered in the compact was the focus on continuous quality improvement from the perspective of the patient. The associated responsibuties for NHS

formoving the NHS fowable approach oving the NHS forward in terms organisations and senv, fe regulatory adjust processes to put patients fistan develop trusting and respectfut tross organisational relationships.

\section{Behind the Research}

\section{Dr Nicola Burgess} Virginia Mason Production System, even
Research Objectives

Nicola Burgess' main research interests include quality mprovement in healthcare, service improvement, patient safety, organisational learning and knowledge

\section{Detail}

Nicola Burgess

perations Group, Warwick Business School rsity of Warwick

Coventry, UK

Nicola Burgess is Reader in Operations Management at Warwick Business School, University of Warwick. Nicola has led multiple research projects within a healthcare context. Her work has been published in world leading journals including Human Resource European Journal of Operations Research and British Journal of Management.

Funding

The Health Foundation, NHS England, NHS Improvement

\section{Collaborators}

Professor Graeme Currie Professor Bernard Crump

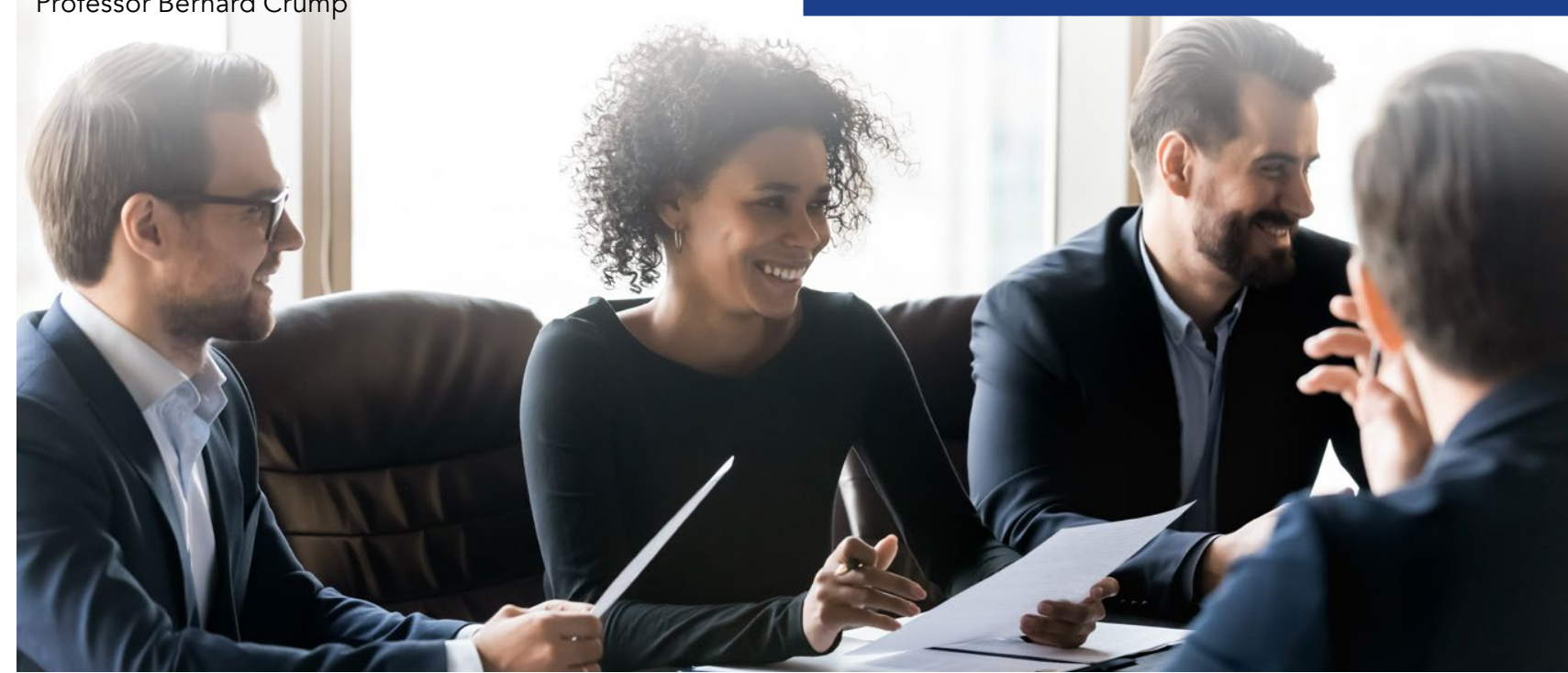

Burgess, N., Currie, G., Crump, B., Richmond, J., and Johnson, M. (2019). Improving together: collaboration needs to start with regulators. BMJ 367:16392. https://do org/10.1136/bmj.16392

\section{Personal Response}

In your opinion, what is the largest barrier to

II Relational authority emerged over time through

mechanisms that fostered trust among all representatives, anchored by a shared goal of developing a sustainable
continuous improvement capability in the NHS. A more relational approach to governance produces interorganisational learning. leanning from each other, learning is far removed from the blame culture that too often erodes staff morale and relatedly, patient safety, in healthcare provider organisations.

This collaboration was underpinned by a 'spirit of partnership' that further benefitted from a significant authority to emerge. The challenge for the NHS and any healthcare system, is how we make time to routinely bing senior healthcare leaders together, to create a protected
relational space for inter-organisational learning.

\section{References} of time from senior NHS leaders that allowed relational 